MONTANA

STATE UNIVERSITY

LIBRARY

\title{
Ferroelectricity Experiment for Advanced Laboratory
}

\author{
Author: V. Hugo Schmidt
}

Copyright (1969) American Institute of Physics. This article may be downloaded for personal use only. Any other use requires prior permission of the author and the American Institute of Physics. The following article appeared in American Journal of Physics and may be found at (URL/link for published article abstract).

Schmidt, V. Hugo. "Ferroelectricity Experiment for Advanced Laboratory." American Journal of Physics 37, no. 4 (1969): 351. doi:10.1119/1.1975572.

Made available through Montana State University's ScholarWorks scholarworks. montana.edu 


\section{AMERICAN \\ JOURNAL \\ of PHYSICS}

\section{Ferroelectricity Experiment for Advanced Laboratory}

V. Hugo Schmidt

Citation: American Journal of Physics 37, 351 (1969); doi: 10.1119/1.1975572

View online: http://dx.doi.org/10.1119/1.1975572

View Table of Contents: http://scitation.aip.org/content/aapt/journal/ajp/37/4?ver=pdfcov

Published by the American Association of Physics Teachers 


\title{
Ferroelectricity Experiment for Advanced Laboratory
}

\author{
V. HUGO SCHMIDT \\ Depariment of Physics, Montana State University, Bozeman, Montana 59715
}

(Received 5 January 1968; revision received 4 November 1968)

\begin{abstract}
An experiment suitable for a junior or senior physics laboratory is described, in which the spontaneous polarization and coercive field for Rochelle salt are measured from the ferroelectric hysteresis loops, over the temperature range $-18^{\circ}$ to $+24^{\circ} \mathrm{C}$ in which this crystal is ferroelectric. The suitability of triglycine sulfate for this experiment is discussed. The apparatus is also useful in demonstrating the relation of capacitance to electrode geometry, and in determining dielectric constants.
\end{abstract}

\section{INTRODUCTION}

Ferroelectric crystals are an interesting class of materials which have a number of practical applications and are receiving more and more attention, although they are usually barely mentioned in the undergraduate physics curriculum. They are similar phenomenologically to ferromagnetic materials in that they exhibit hysteresis loops, spontaneous polarization, and coercive field, so an experiment on ferroelectrics might well be performed in sequence with an experiment on hysteresis in magnetic materials. Rochelle salt is suitable for an advanced laboratory experiment on ferroelectricity because it has two ferroelectric transition temperatures, or Curie points, both near room temperature, so the student can observe the growth and decay of the hysteresis loop with changing temperature. Triglycine sulfate could also be used. It has only one Curie point, at $49^{\circ} \mathrm{C}$, but does not have the storage problems encountered with Rochelle salt. Both crystals are easily obtained, the crystal holder is of simple construction, and the other necessary equipment is generally available in physies laboratories.

\section{THEORY}

A simplified version of the experimental circuit is shown in Fig. 1. The voltage $V_{y}$ across the vertical deflection plates of the oscilloscope is given by

$$
V_{y}=\frac{1}{C} \int I d t=\frac{1}{C} \int \frac{d Q_{x}}{d t} d t=\frac{Q_{x}}{C}
$$

By Gauss' law the free charge $Q_{x}$ on the crystal electrodes is given in mks units by

$$
\begin{aligned}
Q_{x} & =A D=A\left(\epsilon_{0} E+P\right), \\
& =A\left(\epsilon_{0} E+\chi \epsilon_{0} E+P_{f}\right)=A\left(K \epsilon_{0} E+P_{f}\right) .
\end{aligned}
$$

Here $A$ is the electrode area, $\chi$ and $K$ are, respectively, the electric susceptibility and dielectric constant characterizing the linear part of the

FIg. 1. Simplified experimental circuit. This circuit is essentially equivalent to the SawyerTower circuit (Ref. 3,p.5) because $C$ is made large enough so that almost all of the voltage $V_{x}$ appears across the crystal.

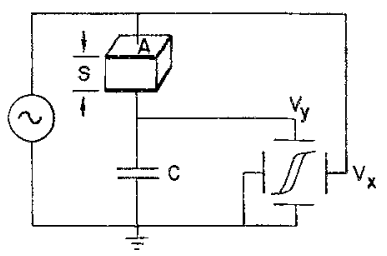

polarization $P$, and $P_{f}$ is the nonlinear part of the polarization associated with the ferroelectric behavior. If $C$ is large enough so that the voltage across the crystal is essentially the voltage $V_{x}$ applied to the horizontal deflection plates, then $V_{y}$ from $\mathrm{Eq}$. (2) can be written as

$$
V_{y}=Q_{x} / C=(A / C)\left(K \epsilon_{0} V_{x} / S+P_{f}\right)
$$

For a linear dielectric $P_{f}$ is zero, and the oscilloscope will display a straight line with slope proportional to the dielectric constant $K$ and the 


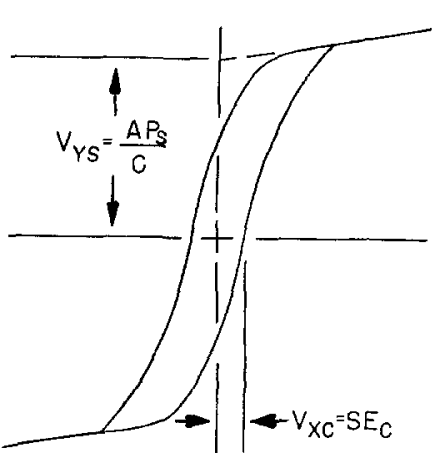

FIG. 2. Hysteresis loop from Rochelle salt at $5^{\circ} \mathrm{C}$. Further discussion of hysteresis loop is given in Ref. 3, p. 4.

capacitance $C_{x}$ of the sample:

$$
d V_{y} / d V_{x}=K \epsilon_{0} A / C S=C_{x} / C .
$$

An oscilloscope trace of the hysteresis loop representing $V_{y}$ VS $V_{x}$ for Rochelle salt is shown in Fig. 2. The straight portions of the loop ending at the points represent times at which $P_{f}$ has reached its maximum or saturation values $\pm P_{s}$. The spontaneous polarization $P_{s}$ is found by extrapolating the straight line back to zero applied field, as indicated on Fig. 2. Another parameter of interest is the coercuve field $E_{c}$, which is the applied field necessary to reduce the polarization $P$ to zero. As indicated on Fig. 2, $E_{c}$ is essentially $V_{x} / S$ at the time that $V_{y}$ vanishes.

\section{DESCRIPTION OF APPARATUS}

The experiment consists basically in applying the voltage across the crystal also to the horizontal deflection plates of an oscilloscope, and simultaneously applying a voltage proportional to the integral of the current through the crystal to the vertical plates. This second voltage is almost proportional to the polarization of the crystal, so in a ferroelectric crystal a hysteresis loop representing polarization vs applied field is obtained. For a linear dielectric the loop degenerates to a straight line.

The circuit is shown in Fig. 3. It is a modification of the Sawyer-Tower circuit. ${ }^{1}$ A Variac operating from the 60 -cycle line gives satisfactory results as a power source, but it can be replaced by an audio oscillator to allow observation of the

\footnotetext{
${ }^{1}$ C. B. Sawyer and C. H. Tower, Phys. Rev. 35, 269 (1930).
}

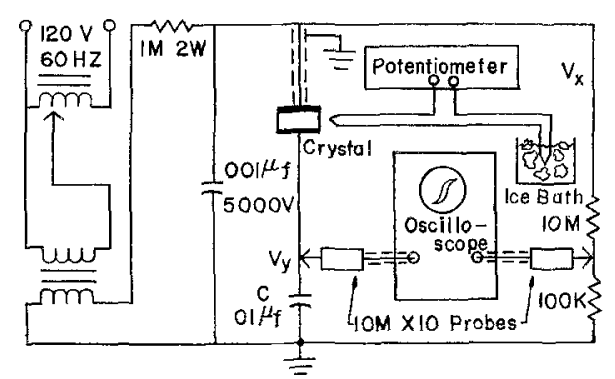

FIG. 3. Circuit for observing ferroelectric hysteresis loops.

change in the hysteresis loop with frequency. The transformer should be able to supply at least $2000 \mathrm{~V}$ peak-to-peak for a crystal of several $\mathrm{mm}$ thickness.

The network attached to the transformer secondarily filters out harmonies, and limits current in case of short circuit. The voltage divider protects the horizontal input probe from excessive voltage; a high-voltage probe could instead be attached directly to the lead going to the crystal. The capacitor should be increased to $0.1 \mu \mathrm{F}$ if larger crystals having contact areas of about $1 \mathrm{~cm}^{2}$ are used.

The mechanical arrangement is illustrated in Fig. 4. The sample holder frame is a strip of brass bent into a $\mathrm{C}$. The lower end holds a screw which adjusts the position of a stand-off insulator to which the shielded high-voltage cable is attached. The upper end holds a feed-through insulator which conducts the current from the crystal to the capacitor $C$. The crystal holder is mounted in

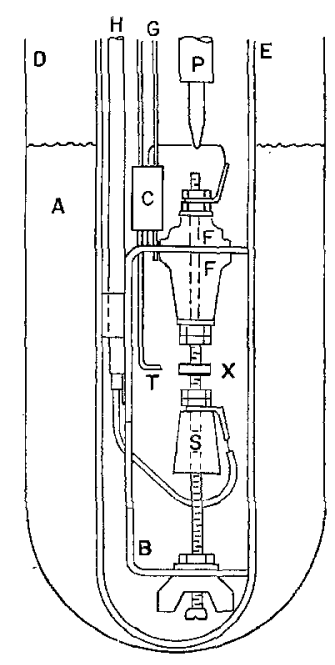

Frg. 4. Full-scale drawing of crystal holder and associated equipment. $A$ is liquid serving as heat bath; $B$ is brass frame serving as ground for $C, G$, and shield of $H ; C$ is $0.01-\mu \mathrm{F}$ capacitor; $D$ is inner wall of Dewar or thermos; $E$ is large test tube; $F$ is feed-through insulator; $G$ is ground lead for probe, which screws into threaded hole in $B$; $H$ is coaxial cable supplying high voltage from transformer to crystal; $P$ is oseilloscope probe connected to vertical (Y) input; $S$ is stand-off insulator; $T$ is thermocouple; $X$ is crystal. 
a large test tube with a cork or stopper having holes for the oscilloscope probe, the high-voltage coaxial cable, the thermocouple wires if used, and the dry-gas inlet.

The dry gas prevents moisture from accumulating on the crystal as it is cooled. Such an accumulation would provide a low-resistance path around the crystal, resulting in incorrect hysteresis loops. This gas can be provided from a compressed gas cylinder, or a thermos or Dewar containing liquid nitrogen or dry ice. Dry gas is not needed if the temperature is kept within a few degrees of the upper Curie point of $24^{\circ} \mathrm{C}$.

Rochelle-salt single crystals can be purchased from Semi-Elements, Inc., Saxonburg, Pa., or can be easily grown using a recipe given by Holden and Singer.2 The ferroelectric $(a)$ axis can be identified from Fig. VII-1 of Jona and Shirane. ${ }^{3}$

The crystal must be cut to shape with a wetstring technique, ${ }^{3}$ or gently rubbed across mediumfine sandpaper, to provide two faces perpendicular to, and remaining faces parallel to, the $a$ axis. It is absolutely necessary to paint or evaporate ${ }^{3}$ electrodes onto the faces perpendicular to the $a$ axis, because if the crystal is merely pressed between metal plates the small air gaps remaining cause serious error due to the crystal's high dielectric constant. Silver paint is available from Du Pont and from Englehard Industries, Inc., Hanovia Liquid Gold Division, East Newark, N. J.

As diseussed in Jona and Shirane, ${ }^{3}$ crystals should be stored in relative humidities between $40 \%$ and $85 \%$. In a dry atmosphere, water of hydration will escape from the surface, leaving a white coat. If this white coat forms under electrodes, the electrodes and coat must be sanded off and new electrodes applied.

\section{PROCEDURE}

A liquid such as light silicone oil which will not freeze at the lower Curie point of $-18^{\circ} \mathrm{C}$ should be used in the thermos or Dewar to provide a heat bath around the test tube. To obtain a temperature above the upper Curie point of $24^{\circ} \mathrm{C}$ at the

2 A. Holden and P. Singer, Crystals and Crystal Growing (Doubleday and Company, Inc., Garden City, N. Y., 1960).

${ }^{3}$ F. Jona ard G. Shirane, Ferroelectric Crystals (The Macmillan Company, New York, 1962). start of the experiment, the liquid should be gently warmed before placing it in the Dewar. It should be remembered that the crystal will decompose at $55.6^{\circ} \mathrm{C}$. To lower the bath temperature, one can insert chips of dry ice or pour in liquid nitrogen through a pyrex funnel. If too much is inserted at one time, a messy boil-over will result. It will take 5 or 10 min for the crystal to reach a new temperature after the bath temperature is altered. Use of a thermocouple near the crystal will provide quite an accurate measure of crystal temperature. A thermometer inside the test tube or in the bath could be used for simplicity, but when the bath is near $-18^{\circ} \mathrm{C}$, the crystal will be a few degrees warmer due to heat leaks along the test tube.

The spontaneous polarization $P_{s}$ and the coercive field $E_{c}$ are obtained from the hysteresis loop as indicated in Fig. 2. The coercive field for Rochelle salt depends on the sample thickness ${ }^{3}$ and maximum applied field. One could also measure the small-signal dielectric constant as a function of temperature, but this measurement requires a high-sensitivity oscilloscope or a preamplifier because the applied voltage must be kept low enough to prevent switching of domains. The onset of domain switching is evidenced by the straight line turning into an ellipse.

Students in our senior laboratory have been able to complete the experiment in an afternoon. They generally have obtained results within a factor of 2 of the published data for spontaneous polarization. ${ }^{3}$

Another material which appears suitable for this experiment is triglycine sulfate (TGS). Jona and Shirane $e^{3}$ give a thorough review of the properties of TGS, including growth methods and graphs of spontaneous polarization and coercive field as functions of temperature. Single crystals can be purchased from Semi-Elements Inc. at a considerably higher price than for Rochelle salt, or they can be readily grown from aqueous solution by evaporation or cooling. Unlike Rochelle salt, TGS can be stored at any humidity. Plates perpendicular to the ferroelectric $b$ axis as required in this experiment can be obtained easily by cleavage along this crystal's only pronounced cleavage plane. TGS is a less interesting but more typical ferroelectric than Rochelle salt because it 
has only one Curie point, which is at $49^{\circ} \mathrm{C}$. Its higher Curie temperature allows omission of the cooling and dry gas provisions needed for Rochelle salt. A heating arrangement, such as a resistor immersed in the liquid bath and driven by a Variac or variable voltage de power supply, must be provided instead.

\section{USE IN MEASURING CAPACITANCE AND DIELECTRIC CONSTANTS}

This device has been used in demonstrations of capacitance as a function of geometry, and measurement of dielectric constants for linear dielec- trics. To measure dielectric constants, two similar rectangular metal plates of area $A$ with nuts soldered on one side are screwed onto the studs. Samples of the dielectrics of thickness $S$ are placed between the plates. The circuit of Fig. 3 is used, and the dielectric constant $K$ is found from Eq. (4). With dielectric absent, the variation of $C_{x}$ with $S$ can be measured, and emergence of edge effects noted as $S$ becomes larger. A set of several cylindrical electrodes with nuts attached has been constructed and used to demonstrate the relation $C_{x}=2 \pi \epsilon_{0} L / \ln \left(r_{2} / r_{1}\right)$ for concentric cylinders.

\title{
Temperature-Dependent, Indirect-Exchange Interaction between Magnetic Ions
}

\author{
M. I. Darby \\ Department of Pure and Applied Physics, University of Salford, Lancashire, England \\ (Received 20 August 1968)
}

\begin{abstract}
Using first-order perturbation theory by analogy with the sereening of a point charge by an electron gas, it is shown how the Ruderman-Kittel-Kasuya-Yosida indirect exchange interaction can be generalized to be temperature-dependent. An approximate expression is obtained for the molecular field in this case, and its application is briefly discussed.
\end{abstract}

\section{INTRODUCTION}

The interaction of the conduction electrons with localized magnetic moments in metals and alloys results in many interesting phenomena in solids. Thus it produces an indirect exchange coupling, the Ruderman-Kitteli-Kasuya ${ }^{2}-$ Yosida $^{3}$ (RKKY) interaction which, because of its long-range oscillatory nature, can give rise to complex-ordered magnetic structures if the density of magnetic ions is sufficiently large. The magnetic properties of the rare-earth metals and alloys can be accounted for in this way. On the other hand, Kondo, ${ }^{4}$ using second-order perturbation theory, has shown that

\footnotetext{
1 M. A. Ruderman and C. Kittel, Phys. Rev. 96, 99 (1954).

2 T. Kasuya, Progr. Theor. Phys. (Kyoto) 14, 45 (1956).

${ }^{3}$ K. Yosida, Phys. Rev. 106, 893 (1957).

${ }^{4}$ J. Kondo, Progr. Theor. Phys. (Kyoto) 32, 37 (1964).
}

the interaction can lead to a term in the resistivity of alloys which has a logarithmic temperature dependence, and which explains the observed low-temperature minima in the resistivity of alloys containing small concentrations of magnetic ions.

We consider only first-order perturbation theory here and hence shall be solely concerned with the RKKY interaction. We will show how it is possible to present the theory in a relatively simple way, by analogy with the screening of a point charge by an electron gas. Moreover, by using this treatment the usual zero-temperature results are readily generalized to be temperature-dependent. We do this here and reproduce a basic result, Eq. (3), first obtained by Baltensperger and de Graaf, ${ }^{5}$

${ }^{5}$ W. Baltensperger and A. M. de Graaf, Helv. Phys. Acta. 33, 881 (1960). 